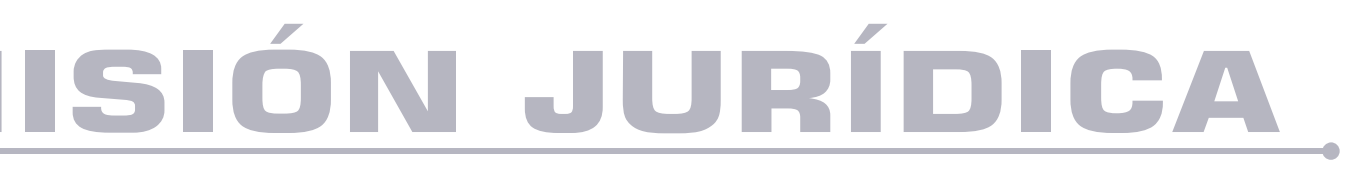

\title{
Historia y desarrollo de la participación de la mujer en al ámbito público en México
}

History and development of women's participation in the public sphere in Mexico

Autor: Luis Antonio Corona Nakamura

DOI: https://doi.org/10.25058/1794600X.1909

\footnotetext{
SP MISIÓN JURÍDICA A 


\title{
HISTORIA Y DESARROLLO DE LA PARTICIPACIÓN DE LA MUJER EN AL ÁMBITO PÚBLICO EN MÉXICO*
}

\author{
History and development of women's participation \\ in the public sphere in Mexico
}

\section{História e desenvolvimento da participação das mulheres na esfera pública no México}

Luis Antonio Corona Nakamura ${ }^{a}$ lacn2004@yahoo.com.mx

Fecha de recepción: 6 de junio de 2020 Fecha de revisión: 30 de junio de 2020 Fecha de aceptación: 1 de julio de 2020

\section{RESUMEN}

DOI: https://doi.org/10.25058/1794600X.1909

Para citar este artículo:

Corona Nakamura, L. A. (2021). Historia y desarrollo de la participación de la mujer en al ámbito público en México. Revista Misión Jurídica, 14(20), 86 -98.

El artículo tiene como objetivo principal analizar la importancia de la paridad de género en México. Para lograrlo se observa el contexto histórico de la violencia en contra de la mujer en el ámbito público internacional y nacional; además, se estudian sus conceptos fundamentales a efecto de entender qué es el género, la paridad y cuál la importancia de su aplicación. Se aporta al campo de estudio un verdadero panorama respecto de la exclusión de la mujer a efecto de ser conscientes de ello y modificar el trato hacia ella, en pro de su beneficio mediante la elaboración y adecuación de normas acordes a la realidad que viven, pues de no ser así, éstas serían ineficaces.

\section{PALABRAS CLAVE:}

Derechos humanos; paridad de género; perspectiva de género; discriminación; igualdad de acceso de oportunidades.

\section{ABSTRACT}

The main objective of this work is to analyze the importance of gender parity in Mexico. To achieve this, the historical context of violence against women in the international and national public sphere

\footnotetext{
* Artículo de reflexión.

a. Profesor de tiempo completo en los programas de licenciatura, maestría y doctorado en Derecho de la Universidad de Guadalajara (Guadalajara, México). Doctor en Derecho por la Universidad Autónoma de Aguascalientes. Licenciado y Maestro en Derecho por la Universidad de Guadalajara. Miembro del Sistema Nacional de Investigadores (SNI nivel I), actualmente director del Instituto de Investigación y Capacitación en Derechos Humanos de la Comisión Estatal de Derechos Humanos Jalisco
} 
is observed, studying the fundamental concepts in order to understand what gender and parity are and the importance of their application. The field of study provides a true panorama with regards to the exclusion of women in order to be aware of it and modify the treatment towards them, for their benefit through the elaboration and adaptation of norms according to the reality they live in since otherwise, they would be ineffective.

\section{KEY WORDS:}

Human rights; gender parity; gender perspective; discrimination; equal access to opportunities.

\section{RESUMO}

O objetivo principal do artigo é analisar a importância da paridade de gênero no México. Para tanto, observa-se o contexto histórico da violência contra a mulher na esfera pública internacional e nacional; Além disso, seus conceitos fundamentais são estudados para entender o que é gênero, paridade e qual a importância de sua aplicação. Proporciona-se ao campo de estudo um verdadeiro panorama da exclusão da mulher para conhecê-la e modificar o tratamento a ela, em prol de seu benefício através da elaboração e adaptação de normas de acordo com a realidade em que vivem, caso contrário. eles seriam ineficazes.

\section{PALAVRAS-CHAVE}

Direitos humanos; paridade de gênero; perspectiva de gênero; discriminação; igualdade de acesso às oportunidades.

\section{INTRODUCTION}

La violencia contra de la mujer no es nueva en la sociedad, a pesar de contar con derechos adquiridos a lo largo de su lucha por los mismos, las mujeres y los hombres no se encuentran en igualdad real de condiciones. Dicho lo anterior, se analizará el ámbito público respecto a la participación de las mujeres en los espacios públicos del país, desde la interrogante, ¿realmente entendemos cómo lograr una igualdad real?

Para obtener respuesta, resulta necesario conocer y entender la carga histórica que tienen las mujeres a efecto de entender su lucha de derechos por ser independientes y actoras de su propia vida.

A efecto de eliminar las desigualdades, surgen medidas afirmativas entre ellas las cuotas de género; sin embargo, ante la violación de las instituciones respecto a las mismas, resulta necesario optar por la paridad de género, una medida permanente que busca lograr la igualdad entre mujeres y hombres, buscando resolver dicha problemática.

Es por ello que para entender realmente que es la paridad de género, se estudiarán conceptos tales como derechos humanos, derechos fundamentales, discriminación, personas en situación de vulnerabilidad, género, medidas afirmativas y su relación respecto a la perspectiva y cuotas de género, sin dejar a un lado la noción de justicia.

\section{DERECHOS HUMANOS}

En la actualidad, los derechos humanos son tendencia social, mismos que se encuentran consagrados en la Declaración Universal de los Derechos Humanos (DUDH) cuyos ideales son la libertad y la justicia, el reconocimiento de la dignidad y valor de las personas, la igualdad de derechos entre hombres y mujeres con la finalidad de promover el progreso de la sociedad y elevar su nivel de vida; derechos protegidos por un régimen de Derecho (DUDH, 1948, p 34).Para la Organización de las Naciones Unidas (ONU), los derechos humanos son "derechos inherentes a todos los seres humanos, sin distinción alguna de raza, sexo, nacionalidad, origen étnico, lengua, religión o cualquier otra condición" (s.f.).

\subsection{Derechos fundamentales}

Definidos los derechos humanos, y retomando el preámbulo de la DUDH, se establece que dichos derechos deben ser protegidos por un régimen de Derecho. Gregorio Peces-Barba (2004), pionero de los derechos fundamentales, manifestaba que el concepto de derechos humanos es el término más común; sin embargo, representan un anhelo que alude a una pretensión moral y a un sistema de derecho positivo (p. 20).

Para este jurista y catedrático español, el concepto de derechos fundamentales es integrador y claro, superando tanto al iusnaturalismo 
como el positivismo ya que para él, los derechos fundamentales abarcan tanto la moralidad como la juridicidad, respondiendo al porqué y el para qué de los derechos fundamentales (p. 29) ya que de no existir juridicidad, los derechos fundamentales carecerían de eficacia, convirtiéndose en consejos o buenas intenciones, resultando necesario que los mismos se encuentren establecidos en la ley para que el Estado exija su respeto (pp. 31, 32 y 38).

\subsection{Discriminación por género}

Desde la antigüedad las mujeres no han sido tomadas en serio, a pesar de su lucha constante por el reconocimiento de sus derechos, siguen siendo excluidas, existiendo así desigualdad de condiciones entre hombres y mujeres. Es cierto que esta exclusión puede afectar a ambos; sin embargo, el impacto que tiene hacia ellas se potencia tanto por la carga histórica como por la existencia de una doble o triple discriminación por cuestión de género, origen, edad, opinión política, entre otros (INADI, 2016, p. 6).

El Comité de los Derechos Humanos del Sistema de UN (1989) define la discriminación como la distinción, exclusión, restricción o preferencia que se base en determinados aspectos que tenga como finalidad anular o menoscabar el reconocimiento, goce o ejercicio en condiciones de igualdad los derechos humanos y libertades de las personas (p. 2). No obstante, dicho concepto se limita al no contemplar como motivo de discriminación la cuestión de género, resultando ser una definición que limita el tema que nos compete.

Por otra parte, el Consejo Nacional para Prevenir la Discriminación en México CONAPRED la define como "una práctica cotidiana que consiste en dar un trato desfavorable o de desprecio inmerecido a determinada persona o grupo, que a veces no percibimos, pero que en algún momento la hemos causado o recibido" (s.f.).

Analizando el concepto, vemos que trata de justificar la discriminación al señalar textualmente que a veces no la percibimos, pero que en algún momento la hemos causado o recibido, situación que visibiliza la realidad de la sociedad al no saber si una persona lleva a cabo actos discriminatorios o si ha sido discriminada.
La Convención sobre la Eliminación de todas las formas de Discriminación contra la Mujer CEDAW de 1979, establece que la discriminación contra la mujer es la distinción, exclusión o restricción basada en el sexo que tenga como finalidad menoscabar o anular el reconocimiento, goce o ejercicio de la mujer (p. 24).

La protección y reconocimiento real de la igualdad y libertad de las mujeres resulta necesaria, ya que las mismas como colectivo se encuentran entre las personas en situación de vulnerabilidad, considerada como aquella situación en la que el Estado debe de satisfacer el respeto y garantía de derechos de las personas en razón a sus necesidades, condiciones y situaciones particulares (Corte Interamericana de Derechos Humanos CIDH, 2006, p. 31).

Aunado a lo anterior, Eduardo San Miguel Aguirre (2000, p. 77) señala que la vulnerabilidad hace referencia a la capacidad de respuesta individual o colectiva frente a una situación determinada, la cual se origina a partir de la reunión de factores internos y externos que, al combinarse, disminuyen o anulan la capacidad que tienen las personas para enfrentar una situación determinada que le ocasione daño. Para él, los factores internos forman parte de las características propias del individuo, tales como el género, mientras que los factores externos están ligados al contexto social.

Amelia Valcárcel (1997) refiere que las mujeres no tienen individualidad por sí mismas, siempre son referidas como la madre, la hermana, la hija, la esposa de alguien que si tiene individualidad (p.30). A su vez, afirma que las mujeres deben de ser libres para poder acceder a la igualdad, ya que las desigualdades son déficits de la libertad.

\section{ANTECEDENTES HISTÓRICOS RESPECTO A VIOLENCIA EN CONTRA DE LA MUJER EN EL ÁMBITO PÚBLICO}

\subsection{A nivel internacional}

Nuria $\operatorname{Varela}^{1}$ (2019) señala que en el siglo XVIII, durante la Ilustración nació el feminismo,

1. Experta en feminismo y género, cuenta con Masters en estudios interdisciplinares de género y en Género y Políticas de Igualdad entre Mujeres y Hombres, por la Universidad Rey Juan Carlos. Véase más en http://nuriavarela.com/acerca-de-nuriavarela/. En su libro Feminismo para principiantes desarrolla los antecedentes y las olas en las que divide el feminismo, lectura 
movimiento social, teoría y práctica política que surgió cuando las y los revolucionarios e ilustrados franceses comenzaron a defender las ideas y principios de igualdad, estableciendo el feminismo como principio: las mujeres son las actoras de su propia vida y no deben de compararse en relación a los hombres, manifestando claramente que la palabra hombre no resulta ser una palabra neutra a utilizar como sinónimo de persona (pp. 13, 14 y 17).

La Declaración de Independencia de Estados Unidos de América y la Declaración de los Derechos del Hombre en Francia, fueron documentos importantes en cuyo contenido se encontraba la libertad, la búsqueda de felicidad, la igualdad jurídica y la libertad personal. Sin embargo, Nuria Varela enfatiza que dichos derechos eran solo para los hombres, y por lo tanto, los derechos de las mujeres no fueron reconocidos, algunas de ellas tenían la idea de que los derechos eran para hombres y mujeres, pero al defender sus ideales, terminaron en la guillotina (pp. 13, 14 y 28).

Posteriormente surgieron los Cuadernos de Quejas, refiriendo Olivia Blanco (2000) que para Olympe de Gouges ${ }^{2}$, las mujeres en 1789 hicieron oír sus voces por escrito participando en la vida política, manifestando las esperanzas de cambio que tenían en la época (p. 38).

En uno de los escritos, una mujer que firmaba como Madame B.B. ${ }^{3}$ señaló que los representantes deben tener los mismos intereses que los representados, ejemplificando que un noble no podía representar a un plebeyo, ni el plebeyo a un noble, y que siguiendo esa lógica, un hombre no podía representar a la mujer, ya que a las mujeres debían de permitírseles representarse por sí mismas (Alonso 1997, p. 2).

En 1789 se proclamó la Declaración de los Derechos del Hombre y del Ciudadano DDHC en

que servirá de apoyo para antecedentes relevantes al tema en cuestión.

2. Nombre que ha sido escrito en diversos artículos como Olimpia de Gouges. Su nombre verdadero era Marie Gouzes. Nació en Montauban, Francia en 1748, quien en palabras de Gloría Ramírez formó parte de las "heroínas del feminismo revolucionario"'” Véase más en https://catedraunescodh.unam. $\mathrm{mx} / /$ catedra/catedra/materiales/u1_cuaderno2_trabajo.pdf

3. Realizando una búsqueda, Madame B.B. fue el anónimo utilizado de quien escribió en el respectivo Cuaderno de Quejas. Véase más en http://www.acuedi.org/ddata/7270.pdf
Francia, declarando que los hombres nacen libres e iguales en derechos (p. 535). Al analizar tanto el título de la DDHC como su contenido, se observa que la situación para las mujeres no ha cambiado, siguen siendo excluidas.

Olympe de Gouges publicó la réplica feminista de la DDHC bajo el nombre de la Declaración de los Derechos de la Mujer y de la Ciudadana, en cuyo contenido señalaba (Ramírez, 2015, pp. 10 y 11):

\section{Artículo I}

La mujer nace libre y goza igual que el hombre de los derechos. Las distinciones sociales sólo pueden fundarse en la utilidad común.

\section{Artículo IV}

La libertad y la justicia consiste en devolver todo lo que le pertenece al otro; así el ejercicio de los derechos naturales de la mujer no tienen más límites que la tiranía perpetua que el hombre le impone. Esos límites deben de ser reformados por las leyes de la naturaleza y de la razón.

\section{Artículo VI}

La ley debe ser la expresión de la voluntad general. Todas las ciudadanas y ciudadanos tienen el derecho de participar personalmente, $o$ por medio de sus representantes en su formación. Ésta debe ser la misma para todos: todas las ciudadanas $\mathrm{y}$ todos los ciudadanos, siendo iguales ante sus ojos, deben de ser igualmente admisibles a todas las dignidades, puestos y empleos públicos, según sus capacidades, y sin otras distinciones que aquellas de sus virtudes y sus talentos. (sic)

Varela (2019) refiere que en 1848, Elizabeth Cady Stanton convocó a hombres y mujeres para hablar de los derechos de las mujeres en un pueblo cerca del estado de Nueva York, resultando así la Declaración de Seneca Falls, en cuyo contenido destaca que todas las leyes que impidan que las mujeres ocupen en la sociedad la posición que su conciencia les dicté o las situé en una posición inferior al hombre, no tiene fuerza ni autoridad (pp. 46, 47, 48, 359 y 360).

A partir de los años setenta, el feminismo utilizó el lema "lo personal es político", haciendo énfasis en que los temas de las mujeres también 
forman parte de la política, surgiendo una división del feminismo de acuerdo a las necesidades propias de las mujeres, naciendo el feminismo institucional del cual la autora señala que fue consecuencia de las conferencias internacionales de la mujer por la ONU, así como la llegada de mujeres a la política y los reclamos políticos de las mujeres a los distintos gobiernos (, pp. 116 y 117).

San Miguel (2000) señala que el feminismo institucional es diferente en cada país, pero tienen como objetivo en común el aceptar cambios radicales dentro del sistema del Estado (pp. 25 y 26).

La ONU decretó el año de 1975 como el año internacional de la mujer en razón a las aportaciones de las mujeres a la vida política, social, económica de sus países, resultando necesario reforzar el reconocimiento del principio universal de igualdad entre hombres y mujeres, enfatizando que los Estados debían de adoptar medidas para garantizar los derechos de ellas (ONU, 1975,p. 73).

Llegado el año de 1975, en México se celebró la primer Conferencia Mundial de la ONU sobre la Mujer, definiendo el plan de acción mundial para los objetivos del año internacional de la mujer (ONU Mujeres, s.f.).

Varela manifiesta que muchas de las mujeres que acudieron eran esposas de jefes de estado, mismas que promovieron intereses políticos propios y no los derechos de las mujeres motivo de reunión (p. 124).

\section{2. Ámbito nacional}

Cuando México era una colonia, las posibilidades de las mujeres eran pocas, su destino era casarse, ingresar al convento o quedarse en casa (Instituto Nacional de Estudios Históricos de la Revolución Mexicana INEHRM, 1995, pp. 6 y 7). En el siglo XIX las mujeres trabajaron fuera del hogar impartiendo clases en escuelas exclusivas para niñas, en el servicio doméstico, en fábricas de hilados, tejidos, puros, cigarros, o en su hogar haciendo costuras (pp. 7 y 8), resultando ser unas de las pocas aspiraciones que podían tener.

Las mujeres han participado en las etapas históricas de México. Por ejemplo, en la Independencia de México participó Josefa Ortiz de Domínguez y Leona Vicario, personajes distinguidas que lucharon con los insurgentes, mientras que en la Reforma, Margarita Maza lo fue en su época; y finalmente: otro ejemplo, durante la Revolución Mexicana fue Hermila Galindo Acosta, colaboradora de Venustiano Carranza, una de las primeras y más importantes feministas en México (INEHRM, 1995, pp. 10 y 12).

En 1916 se celebró el Primer Congreso Feminista en Mérida, Yucatán, espacio de reflexión de los derechos de las mujeres, entre ellos la igualdad de condiciones con los hombres, buscando así la participación femenina en los órganos de representación política (Alejandre \& Torres, 2016, p. 60).

Durante el Congreso Constituyente de 19161917, Hermelinda Galindo solicitó la aprobación del derecho al voto de las mujeres; sin embargo, dicho Congreso rechazó su propuesta, ya que para sus integrantes, pocas mujeres estaban preparadas para ejercerlo (INEHRM, 1995, p. 33). La primera mujer mexicana que participó como candidata a diputada fue Hermelinda Galindo, postulándose por el Distrito $\mathrm{V}$ de la Ciudad de México, pero no fue electa (Valles s.f., pp. 11 y 12). Otro ejemplo es Elvia Carrillo Puerto, que en 1924 fue la primera diputada electa en el estado de Yucatán; sin embargo, duró poco tiempo en funciones, pues debido a amenazas de muerte dejó el cargo y se marchó a Ciudad de México en busca de protección (López, 2017).

Tanto el Congreso Constituyente de 1856$1857^{4}$ como en el de la CPEUM de 1916-1917 ${ }^{5}$, las personas que participaron en su elaboración y discusión fueron diputados, hombres en su totalidad, ninguna mujer participó o dio su opinión en cuanto a su contenido y redacción. Con ello se acredita que la igualdad de conformidad a lo establecido en la CPEUM no resulta ser una igualdad real, dejando a un lado los derechos de las mujeres, entre ellos, la participación igualitaria para el acceso de oportunidades en cargos públicos del país.

El Código Civil para el Distrito y Territorios Federales de 1928 en su artículo 169 señalaba que

4. De conformidad al diario de debates contenido de la Historia del Congreso Estraordinario (sic) Constituyente de 1856 y 1857 de Francisco Zarco.

5. De conformidad al diario de los debates del Congreso Constituyente 1916-1917 de los Estados Unidos Mexicanos de 1960. 
la mujer podía desempeñar un empleo o profesión siempre y cuando no perjudicará el cuidado del hogar. No obstante, dicha legislación contemplaba que si el marido consideraba que ello afectaba a las necesidades del hogar, podía oponerse a que la mujer realizara dichas actividades (Poder Ejecutivo, 1928, p. 45). En dicha situación se observa como el Estado mexicano reconocía que la mujer podía ejercer una profesión siempre y cuando su esposo lo considerará prudente, dicho derecho y decisión no dependía de ella misma, si no de alguien más.

En los discursos políticos de los hombres que aspiraban a ser presidentes de la República, entre ellos Lázaro Cárdenas y Miguel Alemán, prometían el voto a las mujeres; sin embargo, ninguno de ellos lo cumplió a nivel federal ${ }^{6}$.

Miguel Alemán, el 12 de febrero de 1947, reconoció a las mujeres el derecho a votar y ser votadas en las elecciones municipales en igualdad de condiciones que los hombres, reformando así la CPEUM en su artículo 115 (Congreso de la Unión, p. 3); sin embargo, algunos Estados de la República ya lo habían reconocido (González s.f., pp. 15, 19, 20), reforma que solo amplió el alcance del derecho de las mujeres a votar y ser votadas en los Estados que no lo contemplaban.

El 17 de octubre de 1953 México avanzó normativamente al reconocer a las mujeres como ciudadanas de la República en el artículo 34 constitucional (Congreso de la Unión, p. 2).

\section{Discrepancia}

Al ser consideradas como ciudadanas, de conformidad al artículo 35 constitucional, adquirieron a su vez el derecho a votar y ser votadas en cualquier cargo de elección popular, su derecho al voto y su participación política se amplió de lo municipal a lo federal (SCJN, s.f., p. 21), emitiendo su voto por primera vez en las elecciones del año 1955 (Instituto Nacional para el Federalismo y el Desarrollo Municipal, 2019).

No obstante, Manuel González O. manifiesta que el voto a las mujeres fue concedido por el hombre, no fue reconocido como su derecho. ya que ve el derecho del voto a las mujeres como una concesión de Ruiz Cortines, presidente electo de

6. Ello se puede observar al analizar los relatos que realiza Manuel González Oropeza. la República Mexicana, resultando su triunfo una condición para que las mujeres tuvieran derechos políticos (s.f., pp. 21 y 22).

Hasta el 31 de diciembre de 1974, México en su CPEUM reconoció en el artículo $4^{\circ}$ la igualdad del hombre y la mujer ante la Ley (Poder Ejecutivo, 1974, p. 2).

\section{GÉNERO}

Para Rosa Cobo Bedia el concepto de género nació a partir de la idea de que lo femenino y lo masculino son construcciones sociales, no naturales ni biológicas (1995, p. 1).

Varela (2019) refiere que son las obligaciones, comportamientos, pensamientos, capacidades que se le han exigido a la mujer por ser mujer, precisando que sexo y género no son sinónimo, ya que el sexo se refiere a las diferencias físicas y el género a las normas y conductas asignadas a hombres y mujeres (p. 181).

La Organización Mundial de la Salud lo define como las funciones, comportamientos, actividades y atributos que cada sociedad considera apropiados para los hombres y las mujeres, generando desigualdad entre ellos, favoreciendo así a uno de estos (s. f).

\subsection{Techo de cristal}

La sociedad ha creído que los hombres tienen que ser líderes, obteniendo así los cargos de alto mando mientras que las mujeres desempeñan puestos administrativos, fomentando así el techo de cristal.

Amelia Valcárcel (1997) señala que todo poder organizacional es de libre concurrencia o de cooptación. En la primera de ellas, se accede a través de las habilidades y la experiencia; mientras que en la segunda no se juzga ni la habilidad ni la experiencia, si no la adecuación de la persona vinculada por la influencia (p. 98). Para ella, el techo de cristal es una expresión que se designa a las prácticas que dan como resultado que las mujeres no sean tomadas en cuenta por los sistemas de cooptación (p. 98), impidiendo así su acceso a puestos determinados en una organización.

Raquel Osborne (2005) refiere que el techo de cristal es un tope invisible que impide a las mujeres llegar al terreno público; sin embargo, 
señala que a través de los resultados diferenciales se mide la discriminación hacia las mismas, resultados que justifican la adopción de acciones positivas $^{7}$, entre ellas la paridad (p. 163).

\subsection{Medidas afirmativas}

Varela (2019) opina que las medidas afirmativas tienen como objetivo eliminar barreras y facilitar la participación de las mujeres tratando de manera desigual lo desigual para conseguir un equilibrio (p. 190). Si las mujeres no están en igualdad de condiciones que los hombres, las instituciones tienen la obligación de lograr, principalmente con leyes, que el ser mujer no sea una desventaja para asegurar una competencia real y efectiva (Corona, 2019, pp. 5 y 8).

\subsection{Perspectiva de género}

La perspectiva de género es una categoría analítica que toma los estudios teóricosacadémicos del feminismo para cuestionar los estereotipos $\mathrm{y}$, a partir de ello, elaborar nuevos contenidos que incidan en la sociedad respecto a la igualdad y la equidad, permitiendo reconocer que históricamente $y$ en la actualidad las mujeres han tenido oportunidades desiguales e inequitativas (Fondo de las Naciones Unidas para la Infancia UNICEF, 2017, pp. 14 y 15).

Por otra parte, la Organización Internacional del Trabajo la define como el proceso de valorar las acciones que se planifiquen tanto para hombres como para mujeres, observando y anticipando las condiciones y obstáculos que se pueden encontrar para lograr la equidad (s.f.).

El Centro Nacional de Equidad de Género y Salud Reproductiva dependiente de la Secretaría de Salud en México, señala que el hablar de perspectiva de género es tener en cuenta tanto el lugar como el significado, atribuciones e ideas que la sociedad da al hombre y a la mujer tomando en cuenta la diferencia sexual (2010, p. 17).

\subsection{Cuotas de género}

El Instituto Interamericano de Derechos Humanos IIDH y el Tribunal Electoral del Poder Judicial de la Federación TEPJF definen las cuotas de género como la acción afirmativa que establece un número o proporción de la totalidad de espacios o cargos que deben ser obligatoriamente

7. También llamadas acciones o medidas afirmativas. ocupados por un sector discriminado de la sociedad (2017, p. 227).

En la década de los noventa, países de América Latina comenzaron a adoptar medidas afirmativas a través de las cuotas de género, particularmente en sus cámaras legislativas (Archenti, 2011, p. 9).

El primer país del mundo en establecer las cuotas de género fue Argentina en 1991 bajo la denominación de cupo femenino (IIDH et al, 2017, pp. 230 y 231). Posteriormente, México y Paraguay adoptaron las cuotas en 1996, mientras que el Estado Plurinacional de Bolivia, Brasil, Costa Rica, Ecuador, Panamá, Perú y República Dominicana la incorporaron a su normativa en 1997, Honduras la adoptó en el 2000 y Uruguay en el 2009 (Archenti, 2011, p. 16).

Analizado lo anterior, queda claro que las cuotas de género fueron aplicadas al ámbito electoral a efecto de contrarrestar la discriminación de las mujeres al acceso a puestos de elección popular (IIDH et al, 2017, p. 227); sin embargo, en México las cuotas de género no fueron aplicadas en Poderes o ámbitos diversos.

\subsection{Paridad de género}

La noción de paridad nació en Europa mediante la democracia paritaria, mencionada también en Estrasburgo por el Consejo de Europa en 1989 señalando que la igualdad entre hombres y mujeres es cuestión política (Varela, 2019, pp. 194 y 195).

En Atenas, el 3 de noviembre de 1992, la primera Cumbre Europea "Mujeres en el Poder" promulgó la Declaración de Atenas, que establece lo siguiente (Congreso de los Diputados, 1993, p. 1):

PORQUE la igualdad formal y real entre mujeres y hombres es un derecho fundamental del ser humano.

PORQUE las mujeres representan más de la mitad de la población. La democracia exige la paridad en la representación y en la administración de las naciones.

PORQUE las mujeres constituyen la mitad de las inteligencias y de las capacidades potenciales de la humanidad y su interpretación en los puestos de decisión constituye una pérdida para el conjunto de la sociedad. 
PORQUE una participación equilibrada de mujeres y hombres en la toma de decisiones puede generar ideas, valores y comportamientos diferentes, que vayan en la dirección de un mundo más justo y equilibrado tanto para las mujeres como para los hombres.

PORQUE la infra-representación de las mujeres en los puestos de decisión impide asumir plenamente los intereses y las necesidades del conjunto de la sociedad.

PROCLAMAMOS la necesidad de alcanzar un reparto equilibrado de los poderes públicos y políticos entre mujeres y hombres.

REIVINDICAMOS la igualdad de participación de las mujeres y de los hombres en la toma de decisiones públicas y políticas.

DESTACAMOS la necesidad de realizar modificaciones profundas en la estructura de los procesos de decisión con el fin de asegurar dicha igualdad. (sic)

La paridad es igualdad, ya no es una acción afirmativa como las cuotas de género, es una medida permanente, un principio constitucional que busca la igualdad sustantiva entre hombres y mujeres para lograr la inclusión de estas últimas en espacios de decisión pública (Bonifaz, s.f., p. 1).

El Sistema de Información Legislativa define a la paridad de género como un principio para garantizar la igualdad de acceso a puestos de representación política entre mujeres y hombres asegurando la participación igualitaria en los mismos (s.f.). Dicha definición se encuentra enfocada en los puestos de representación política, pero conforme a la naturaleza del significado de la paridad, no es propia del ámbito político. Si bien es cierto que México adoptó dicho principio en el ámbito político, su aplicación no es exclusiva al mismo.

La paridad es una estrategia orientada a combatir la discriminación contra las mujeres que histórica y estructuralmente las mantuvo al margen de los espacios públicos, resultando ser un principio que debe aplicarse en el poder ejecutivo, legislativo y judicial, así como en los organismos autónomos (IIDH et al, 2017, pp. 803 y 804).

Raquel Osborne (2005) manifiesta que al hablar de paridad se piensa en las decisiones políticas tradicionales, respecto a la igualdad de oportunidades entre hombres y mujeres, desde ya el comienzo es difícil, pues se parte desde una desigualdad real, si se aplica la igualdad como se encuentra plasmada en la ley, la diferencia entre hombres y mujeres seguirá aumentando con el transcurso del tiempo (p. 167).

\section{JUSTICIA COMO EQUIDAD}

Como señala Nuria Varela (2019), el espíritu del feminismo es la teoría de la justicia, misma que va transformando las relaciones entre hombres y mujeres, buscando que los seres humanos quieran y vivan como quieran ser, sin un destino marcado por el sexo con el que nacieron (p. 20).

Las mujeres no decidieron ser mujeres y ser mujer no es razón determinante para elegir o ejercer una profesión, ni el cómo quieran vivir, teniendo como obligación las instituciones el reducir las desventajas cuando las mujeres tengan un trato desigual por el simple hecho de serlo (Corona, 2019, p. 5).

Las mujeres no decidieron ser mujeres y ser mujer no es razón determinante para elegir o ejercer una profesión, ni el cómo quieran vivir, teniendo como obligación las instituciones el reducir las desventajas cuando las mujeres tengan un trato desigual por el simple hecho de serlo (Corona, 2019, p. 5).

Lo anterior se encuentra apoyado por la Teoría de la Justicia de John Rawls en la cual hace referencia a la "lotería natural"; consiste en que ninguna persona pidió ni decidió nacer con las características físicas con las que cuenta, en el caso en concreto, ser hombre o mujer, y a su vez, no decidió vivir en una sociedad en el que todo está arbitrariamente decidido (2019, p.3).

Al respecto, Roberto Gargarella (1999) manifiesta que Rawls sostiene que no tiene sentido reprocharle a la naturaleza si es justa o no con las personas, lo que debería de evaluarse es la justicia o injusticia del sistema institucional que procesa los hechos de la naturaleza (p. 41).

La justicia puede ser vista y utilizada desde el punto de la equidad. Para Rawls (2015), la justicia como equidad está pensada para las sociedades democráticas cuyos principios responden a un sistema equitativo, justicia que trata de relacionar los valores y la aplicación de estos en las instituciones (pp. 69 y 70). 
Para el autor, existen dos principios de justicia: el primero de ellos, es que todas las personas tienen el mismo derecho y libertades básicas, y, por lo tanto, cada persona tiene derechos con carácter irrevocable, principio que es aplicable a la Constitución en cuyo contenido se encuentran las libertades. ya que se institucionalizan a través del poder constituyente. Rawls da como ejemplo el derecho a ocupar cargos, derecho que debe institucionalizarse de manera adecuada tanto en la Constitución y los procedimientos específicos para modificar la misma protegiendo su esencia, ejemplo que resulta adecuado para el tema a desarrollar (2015, p. 73, 77 y 78).

El segundo principio, señala que las desigualdades sociales tienen que satisfacer dos condiciones, que las desigualdades deben de estar vinculadas a cargos abiertos a todas las personas en condiciones de igualdad equitativa de oportunidades y que dichas desigualdades no deben dañar ni perjudicar a las personas menos beneficiosos de la sociedad, ya que para él, dicho principio requiere de una igualdad equitativa de oportunidades y que las desigualdades se rijan por el principio de diferencia (2015, p. 73 y 78).

Para Ralws, las desiguales pueden justificarse siempre y cuando existan ventajas para las mujeres y aceptando las mismas, situación que no es así de acuerdo con los antecedentes históricos antes señalados y que el autor reconoce.

"La equidad es correctiva una vez que sabemos que lo equitativo sería aquello que exigiríamos en condiciones de igualdad", regulando los resultados una vez que las personas son libres en igualdad de oportunidades, la equidad implica la imparcialidad de un reparto equitativo entre la justicia natural y la ley positiva (Corona, 2019, pp. 17, 19 y 22).

\section{CONSIDERACIONES FINALES}

Las mujeres tienen derechos humanos por el simple hecho de ser personas, sin que exista limitante alguno para el acceso a los mismos. De acuerdo a Gregorio Peces-Barba, es importante que dichos derechos se encuentren contenidos en una norma, en derechos fundamentales, ya que al proteger valores como la justicia, libertad e igualdad de condiciones entre mujeres y hombres en los espacios públicos, es necesario que su protección se encuentre contenida en una norma que exija el actuar, el respeto y cumplimiento de la misma por la autoridad, pues de no ser así, los derechos de las mujeres se convertirían en un simple anhelo, una aspiración a alcanzar.

Al analizar el aspecto histórico internacional y nacional, se puede afirmar que las mujeres han sido excluidas a lo largo de la historia y a pesar de los derechos adquiridos, aun no acceden a una igualdad real. Uno de los problemas de discriminación contra las mujeres es por cuestión de género, una construcción social que determina el pensar, hacer y sentir de las mujeres y los hombres. La sociedad espera que la mujer sea femenina mientras que del hombre espera su masculinidad.

En México, poco a poco las mujeres han obtenido derechos, pero no ha sido suficiente, ya que como se ha observado, algunos de los mismos no fueron otorgados por ser derechos inherentes a ellas, involucrando así intereses de otros. Nunca fueron tomadas en cuenta las voces de las mujeres que lucharon por sus derechos.

Es por ello que, a efecto de reducir la discriminación y exclusión de las mujeres en el ámbito público, se adoptan medidas afirmativas, mismas que tienen como finalidad eliminar barreras y así facilitar la participación de mujeres en igualdad de condiciones que los hombres. Entre dichas medidas se encuentran la perspectiva y las cuotas de género, mismas que resultan ser de carácter temporal.

No obstante, su adopción, la situación de las mujeres es desfavorable, resultando necesaria así la adopción de la paridad de género, misma que resulta ser una medida de carácter permanente. La paridad de género tiene como finalidad eliminar la discriminación de las mujeres desde la desigualdad misma a efecto de erradicar su aumento.

Para que las mujeres accedan a igualdad y justicia, es necesario realizar reformas reales a la normativa vigente para lograrlo, que no solo se establezca en una norma, ya que, de ser así, las mujeres no accederían a ello, generando nuevamente su exclusión. La igualdad terminaría siendo solo un discurso más. 


\section{BIBLIOGRAPHY}

- Alejandre R., G. L. y Torres A., E. (2016). "El Primer Congreso Feminista de Yucatán 1916. El camino a la legislación del sufragio y reconocimiento de ciudadanía a las mujeres. Construcción y tropiezos". Estudios Políticos. No. 39. Disponible en http://www.revistas.unam.mx/index. $\mathrm{php} / \mathrm{rep} / \mathrm{article} / \mathrm{view} / 57316 / 50841$

- Alonso D., I. (1997). Las mujeres francesas exigieron el sufragio universal: ¿Lo enseñamos en historia?. Madrid: Clio \& Asociados.

- Archenti, N. (2011). La paridad política en América Latina y el Caribe. Percepciones y opiniones de los líderes de la región. Santiago de Chile: Naciones Unidas. Disponible en https://repositorio.cepal. org/bitstream/handle/11362/5835/1/ S1100674_es.pdf

- Blanco, 0. (2000). Olimpia de Gouges (1748-1793). Madrid: Ediciones del Orto.

- Bonifaz A., L. (s.f.). El principio de paridad en las elecciones: aplicación, resultados y retos. Suprema Corte de Justicia de la Nación. Disponible en https://www.scjn.gob.mx/sites/default/ files/derechos_humanos/articulosdh/ documentos/2016-12/PRINCIPIO\%20 DE\%20PARIDAD.pdf

- Centro Nacional de Equidad de Género y Salud Reproductiva. (2010). Guía para la Incorporación de la Perspectiva de Género en Programas de Salud. Ciudad de México. Disponible en https://www.cndh.org. $\mathrm{mx} /$ sites/all/doc/Programas/VIH/Otra sPublicacionesdeinteresrelacionadosco nelVIH/CENSIDA/GUIA_PERSPECTIVA_ GENERO\%20ssa.pdf

- Cobo B., R. (1995). 10 palabras claves sobre mujer. Madrid: Ed. Verbo Divino. Estella Navarra. Disponible en https:// www.te.gob.mx / gen ero/media / pdf/5d9668bc7465d15.pdf

- Comité de los Derechos Humanos del
Sistema de Naciones Unidas. (1989). No discriminación. 10/11/89. CCPR Observación general 18. (General Comments). Disponible en https:// www.acnur.org/fileadmin/Documentos/ BDL/2001/1404.pdf

- CONAPRED.(s.f.) Discriminacióneigualdad. Disponible en www.conapred.org.mx/ index.php?contenido=pagina\&id=84\&id opcion $=142 \&$ op $=142$

- Congreso de los Diputados. (1993). “1401000013 Declaración institucional por la que se asume la Declaración adoptada en la primera". Boletín Oficial de las Cortes Generales. Disponible en http:// www.congreso.es/public_oficiales/L4/ CONG/BOCG/E/E_250.PDF

- Congreso de la Unión. (1947). "Decreto que adiciona el párrafo primero de la fracción primera del artículo 115 de la Constitución Política de los Estados Unidos Mexicanos".. Diario Oficial de la Federación. Disponible en https://www.dof.gob.mx/nota_to_ imagen_fs.php?cod_diario=199133\&pagin $\mathrm{a}=3 \&$ seccion $=0$

- Congreso de la Unión. (1953). "Decreto que reforma los artículos 34 y 115 de la Constitución Política de los Estados Unidos Mexicanos". Diario Oficial de la Federación. Disponible en https://www.dof.gob.mx/ nota_to_imagen_fs.php?cod_diario $=19932$ 9\&pagina $=1 \&$ seccion $=0$

- Constitución Política de los Estados Unidos Mexicanos. (s.f.). Suprema Corte de Justicia de la Nación. Disponible en http://legislacion.scjn.gob.mx/ Buscador/Paginas/AbrirDocArticulo. a s p $\mathrm{x}$ ? $=/$ U z k 6 PWW U G G brF gW / MQRheVHIlPSxd0V3mtVoZHKrknb/latPn ElfWAoa6jM5QaXXORsmHSUgrfrQOpBK2 IeYQ $==$

- Convención sobre la Eliminación de todas las formas de discriminación contra la mujer. (1979). Nueva York. Disponible en 
https://undocs.org/es/A/RES/34/180

- Corona N., L. A. (2019). La justicia como paridad de género. Ciudad de México: Editorial Porrúa.

- Corte Interamericana de Derechos Humanos. (2006). Caso Ximenes Lopes vs. Brasil, Sentencia de 4 de julio de 2016. Disponible en http://www.corteidh.or.cr/ docs/casos/articulos/Seriec_149_esp.pdf

- Declaración de los Derechos del Hombre y del Ciudadano. (s.f.). Las Constituciones de México. Disponible en http://www. diputados.gob.mx/biblioteca/bibdig/ const_mex/decla_huma.pdf

- Fondo de las Naciones Unidas para la Infancia UNICEF. (2017). Perspectiva de Género. Buenos Aires. Disponible en https://www.unicef.org/argentina/sites/ unicef.org.argentina/files/2018-04/COM1_PerspectivaGenero_WEB.pdf

- Gargarella, R. (1999). Las teorías de la justicia después de Rawls. Un breve manual de filosofía política. Barcelona: Paidós. Disponible en https://www.academia. edu/35303017/_V_Las_teor\%C3\%ADas_ de_la_justicia_despu\%C3\%A9s_de_Rawls Un_breve_manual_de_filosof\%C3\%ADa pol\%C3\%ADtica_1_E_eQ_es_at BULLET_M\%C3\%A9xi_co

- González Oropeza, M. (s.f.). El sufragio de las mujeres. ¿Igualdad política por decreto o por convicción? Disponible en https://www.te.gob.mx/comisiones/ comisiones/ad97d806-efb3-4955-8d90$15 \mathrm{bdfe} 4 \mathrm{cf} 42 \mathrm{~d} / \mathrm{CONFERENCIA} \% 20$ O R O P E Z A \% $206 \% 20$ S E P T \% 20 CHICHUAHUA.pdf

- Instituto Interamericano de Derechos Humanos y Tribunal Electoral del Poder Judicial Federal (IIDH y TEPJF). (2017). Diccionario Electoral. Tomo I, A-K. San José de Costa Rica/Ciudad de México. Disponible en https://www.iidh.ed.cr/ capel/media/1440/diccionario-electoral_ tomo-i.pdf

- Instituto Nacional de Estudios Históricos de la Revolución Mexicana. (1995). Hermila Galindo. Biografías para niños. Ciudad de México. Disponible en https://inehrm.gob.mx/recursos/ BibliotecaBicentenario/JovenesYNinios/ HERMILA_GALINDO.pdf

- Instituto Nacional para el Federalismo y el Desarrollo Municipal. (2019). Conmemoramos 64 años del voto de la mujer en México. Disponible en https:// www.gob.mx/inafed/articulos/64aniversario-del-voto-de-la-mujer-en-unaeleccion-federal-en-mexico

- López, A. (2017). "Elvia Carrillo Puerto, 'La monja roja' que logró el derecho al voto femenino en México". El país. Disponible en https://elpais. com/internacional/2017/12/06/ mexico/1512553573_210132.html

- Organización Internacional del Trabajo. (s.f.) Perspectiva de género y equidad social. Disponible en https://www.oitcinterfor. org/general/perspectiva-g\%C3\%A9neroequidad-social

- Organización Mundial de la Salud. (s.f.). Género. Disponible en https://www.who. int/topics/gender/es/

- Osborne, R. (2005). "Desigualdad y relaciones de género en las organizaciones: diferencias numéricas, acción positiva y paridad". Política y Sociedad. Disponible en https://revistas. ucm.es/index.php/POSO/article/view/ POSO0505230163A/22961

- Peces-Barba, G., Roig, R., \& Barranco A., M. (2004). Lecciones de Derechos Fundamentales . Madrid: Dykinson.

- Poder Ejecutivo. (1974). "Decreto que Reforma y Adiciona los Artículos 40., 5o., 3 o y 123 de la Constitución Política de los Estados Unidos Mexicanos, en relación con la Igualdad Jurídica de la Mujer". Diario Oficial de la Federación. Disponible en https://www.dof.gob.mx/website/ nota_to_imagen_fs.php?codnota $=4737525$ \&fecha $=31 / 12 / 1974 \&$ cod_diario $=204144$ 
- Poder Ejecutivo, Secretaría de Gobernación (1928). "Código Civil para el Distrito y Territorios Federales". Diario Oficial de la Federación. Disponible en http:// legislacion.scjn.gob.mx/Buscador/Paginas/ AbrirDocReforma.aspx?q=9kasjIjnGyd4TgPCc ahnJilOaWmNphyYcRRQD1pSeDnmiEF426NS fS8i4QKDT4j82tzXkhDuChy7WXZzvFJRarxm/ vuWQVatEzmMPQb+kyo=

- Ramírez, G. (2015). La declaración de derechos de la mujer de Olympe de Gouges 1791: ¿Una declaración de segunda clase?Disponible en https://catedraunescodh.unam.mx// catedra/catedra/materiales/u1_cuaderno2_ trabajo.pdf

- Rawls, J. (2015). La justicia como equidad. Una reformulación. Barcelona: Paidós.

- San Miguel A., E. (2000). Gaceta de la Comisión Nacional de los Derechos Humanos. Ciudad de
México Disponible en https://www.cndh.org. $\mathrm{mx} / \mathrm{sites} /$ default/files/doc/Gacetas/119.pdf

- Sistema de Información Legislativa. (s.f.). Paridad de Género. Disponible en http://sil. gobernacion.gob.mx/Glosario/definicionpop. php?ID=277

- Valcárcel, A. (1997). La política de las mujeres. Feminismos. Madrid: Cátedra/Universitat de València.

- Valles R., R. M. (2011). "Hermila Galindo: ideas y acción de una feminista ilustrada". Ciencia Universitaria. Disponible en https://www.uaeh.edu.mx/investigacion/ productos/4962/hermila_galindo_articulo_ para_ciencia_universitaria.pdf

- Varela, N. (2019). Feminismo para principiantes. Barcelona: Penguin Random House. 